\title{
Locked Platting for Distal Femur Fractures, Is it a Good Option?
}

\section{Maged Mohamed Mostafa ${ }^{1 *}$, Ahsan Javaid Butt ${ }^{1}$, Ahmed Refaat Khamis $^{2}$, Atul Bandi ${ }^{3}$ and Adeel Nawab Ditta ${ }^{4}$}

${ }^{1}$ Consultant Orthopedic Surgery, King Hamad University Hospital, Bahrain

${ }^{2}$ Consultant Orthopedic Surgery, Benha University Hospital, Egypt

${ }^{3}$ Orthopedic Registrar, King Hamad University Hospital, Bahrain

${ }^{4}$ Orthopedic SHO, King Hamad University Hospital, Bahrain

*Corresponding Author: Maged Mohamed Mostafa, Consultant Orthopedic Surgery,

King Hamad University Hospital, Bahrain.
Received: August 01, 2020

Published: August 19, 2020

(C) All rights are reserved by Maged Mohamed Mostafa., et al.

\begin{abstract}
Distal femoral fractures are among the challenging injuries to be rigidly fixed. Different implants for internal fixation have been used with varying degrees of success. The aim of this study is to review the results of using the distal femoral locked plate (DFLP) in treatment of different fracture patterns of the distal femur.

Methods: A retrospective study was performed between 2012 and 2018 on 41 patients with distal femur fractures treated by distal femoral locked plate (DFLP) in King Hamad University Hospital (KHUH) of Bahrain. Pritchett rating system was used to assess the functional outcome of those patients. Results: total 41 patients were enrolled in the study. There were 13 males and 28 females. The age ranged between 18 and 94 years with an average of 62.9 years. Thirty four patients were followed up till complete union. The average follow up period was 17.2 months. Functional outcome was excellent in 15 patients, good in 10, fair in 4 and poor in 5 cases. Conclusion: distal femoral locked plate (DFLP) is a reliable implant which offers successful results upon using in treatment of various patterns of distal femur fractures.
\end{abstract}

Keywords: Comminuted; Distal Femur; Internal Fixation; Locked Plates; Outcome

\section{Introduction}

Distal femoral fractures account for $4-6 \%$ of all femoral fractures [1-3]. These fractures are encountered in young adults following high velocity injuries and also in elderly population with osteoporosis $[4,5]$.

Internal fixation with blade plate was the standard recommendation in the 1970s by the AO/ASIF (Association for the Study of Internal Fixation). During the following years other implants were developed as the dynamic condylar screw (DCS) with a 95 degree side plate, condylar buttress plate and intramedullary nails. In the presence of comminution and/or osteoporosis, the goals of stable fixation and early mobilization can be difficult to achieve [6-8]. In the recent decades, new technologies were introduced for fixation of distal femoral fractures like the less invasive stabilization sys- tem (LISS) and the anatomical distal femoral locked plate (DFLP). These implants provide multiple points of fixed angle fixation between the plate and the screws. In theory, this should reduce the tendency of varus collapse and failure of fixation $[9,10]$.

\section{Aim of the Study}

This study was aimed at evaluation of the use of laterally applied distal femoral locked plate in treating various patterns of distal femoral fractures.

\section{Methods}

The study was done in KHUH-Kingdom of Bahrain to review the results of distal femur fractures fixation by DFLP after approval from the ethical committee of the hospital. 
Inclusion criteria included all adults with distal femoral fractures A0/OTA classification types 3.2 and 3.3 [11], all closed fractures and open fractures grade 1 and 2 according to Gustello-Anderson classification [12].

Exclusion criteria were femoral fractures in locations other than the distal third, open fractures grade 3 and femoral fractures in skeletally immature patients. This study included 41 patients with an average age of 62.9 years (18-94 years). Ten patients had AO/ OTA classification type 3.2 fractures while 29 patients had type 3.3, and 2 cases had both type 3.2 and type 3.3 fractures. Five patients had Gustello-Anderson Type-I open fracture and 2 cases had open fracture type-II.

Internal fixation by open technique using a direct lateral approach to the distal femur was used in 35 cases while minimally invasive plate osteosynthesis (MIP0) technique was applied in fixation of 6 fractures. Twenty seven patients $(65.8 \%)$ had associated comorbidities. The most common were diabetes mellitus, ischaemic heart disease, chronic kidney disease and Alzheimer disease.

Twenty four patients had abnormal bone mineral density (BMD) at the time of injury: $16(66.6 \%)$ were osteoporotic and 8 patients $(33.4 \%)$ were osteopenic.

After fracture reduction and restoration of length, comminution at the fracture site was evident in 21 cases (51.2\%). Bone substitute in the form of calcium phosphate granules or cancellous bone allograft was added to 10 fractures (47.6\%).

Plate lengths of 9 and 11 holes were the commonest to be used, applied to 25 fractures (60.9\%). Five holes plate was used to fix 6 uni-condylar fractures (type 3.3.B), 13 holes plate was used in two occasions and 7 holes plate was applied to eight fractures. This study included 12 peri-prosthetic distal femur fractures that were fixed by the same technique (29.2\%).

All the patients followed the same postoperative protocol: suction drain was removed 48 hours after surgery, range of knee motion exercises was initiated on the second postoperative day, both passive and active as tolerated, and partial weight bearing using a Zimmer frame was initiated three weeks after the operation except if the patient's neurological or cognitive condition did not permit for safe ambulation, so those patients were kept in bed and their activity was limited to bed-to-chair assisted transfer. In otherwise neurologically normal patients, full weight bearing was allowed only with radiologically evident callus formation.

IBM SPSS 25.0 statistics software was used for all statistical analysis. Student t-test and Mann-Whitney U-test were used to compute the differences between the groups. Pearson correlation analysis was performed for all bivariate analysis. A p-value of less than 0.05 was considered statistically significant.

\section{Results}

A total of 41 patients were enrolled in this study from May 2012 till May 2018, of these 13 (31.7\%) were males and 28 (68.3\%) were females.

Age ranged between 18 and 94 years with an average of 62.2 years. One patient died before achievement of union and 4 cases were lost during the follow up period. Also, 2 cases (both were peri-prosthetic fractures) had complications; one lady aged 94 years had deep infection with metal failure and after metal removal and debridement, the knee joint was surgically fused. The other case was also a lady aged 68 years had pulling out of the locked plate system and went to non-union but refused revision surgery.

The remaining 34 patients were followed up till complete union within a time range of 3-9 months with an average of 6.7 months.

Detailed analysis of the results showed that the mean healing time of fractures in patients who had abnormal bone mineral density was 6.7 months while it was 5.1 months for fractures in patients with normal bone. The difference was statistically significant ( $p=0.045$ ) (Table 1). The average union time for comminuted fractures (21 cases) was 7.2 months while that for non-comminuted fractures was 5.5 months and also the difference was statistically significant ( $p=0.03$ ); (Table 2). So, both low BMD and comminution of the fracture had a positive relation with union. Both factors increased the time to union.

\begin{tabular}{|l|c|c|}
\hline \multicolumn{1}{|c|}{ BMD } & No. (\%) & $\begin{array}{c}\text { Mean healing time } \\
\text { (months) }\end{array}$ \\
\hline Osteoporosis & $24(71 \%)$ & 6.7 \\
\hline Non-osteoporosis & $10(29 \%)$ & 5.1 \\
\hline
\end{tabular}

Table 1: $\mathrm{p}=0.045$. Significant positive correlation between osteoporosis and healing time.

\begin{tabular}{|l|c|c|}
\hline Comminution & No. (\%) & $\begin{array}{c}\text { Healing } \\
\text { (months) }\end{array}$ \\
\hline Yes & $21(61.7 \%)$ & 7.3 \\
\hline No & $31(38.2 \%)$ & 5.5 \\
\hline
\end{tabular}

Table 2: $p=0.03$. Significant positive correlation was observed between union time and fracture comminution. 
Ten patients had artificial bone graft substitute added into the fracture gap at the time of fixation due to comminution and 11 were fixed without grafting. The average healing time was 7.7 and 7 months respectively and the difference was statistically insignificant.

Ritchette rating system [13] was used to assess the functional outcome of patients (Table 3). The knee movement at the latest follow up ranged between 60 and 130 degrees with an average of 106 degrees. Excellent results were found in 14 patients, good in 10, fair in $4(11.76 \%)$ and poor in 6 cases (17.66\%). So, good and excellent results were reported in 24 patients (70.58\%).

\begin{tabular}{|l|c|}
\hline \multicolumn{1}{|c|}{ Result } & Criteria \\
\hline Excellent & $\begin{array}{r}\text { Full extension; flexion }>110^{\circ} \text {; no deformity } \\
\text { or joint incongruity }\end{array}$ \\
\hline Good & $\begin{array}{c}\text { Full extension; flexion }>90^{\circ} ;<5^{\circ} \text { of varus or } \\
\text { valgus; loss of length }<1.5 \mathrm{~cm} \text {, minimal pain }\end{array}$ \\
\hline Fair & $\begin{array}{c}\text { Flexion of } 75^{\circ}-90^{\circ} ; \text { varus, valgus , or angular } \\
\text { deformity of } 5^{\circ}-10^{\circ} ; \text { mild or moderate pain }\end{array}$ \\
\hline Poor & $\begin{array}{c}\text { Flexion }<75^{\circ} ; \text { valgus, varus, or angular de- } \\
\text { formity }>10^{\circ} ; \text { articular incongruity; frequent } \\
\text { pain requiring analgesics }\end{array}$ \\
\hline
\end{tabular}

Table 3: The Pritchett rating system for distal femoral fractures.

\section{Discussion}

This study looked at the results of using anatomical locked plate to fix distal femoral fractures. Fractures of the femur in this region need special care to avoid various complications that could happen; mainly varus mal-union and non-union [14].

Introduction of the locked plating systems reduces in general the complications encountered with the use of conventional plates. Due to the fixed relation between the screws and the plate, the whole design acts as "Internal External Fixator". However, understanding the biomechanical principles of these plates is essential to prevent generation of non-union [15].

We have only one case of aseptic non-union with implant failure (Figure 1). This happened in a lady 68 years old with peri-prosthetic fracture of distal femur. Review of her immediate postoperative $\mathrm{X}$-ray images showed that the plate was not exactly fitting to the bone with a gap of about 2 millimeter. Also, the plate was off the bone at the upper end in both the anteroposterior and the lateral views. All the screw holes close to the fracture region where filled which increased the stiffness of the construct. 


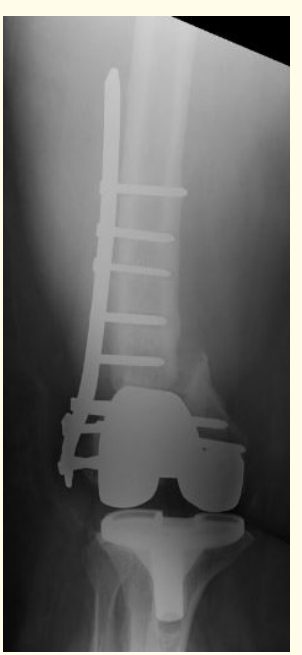

Figure 1d

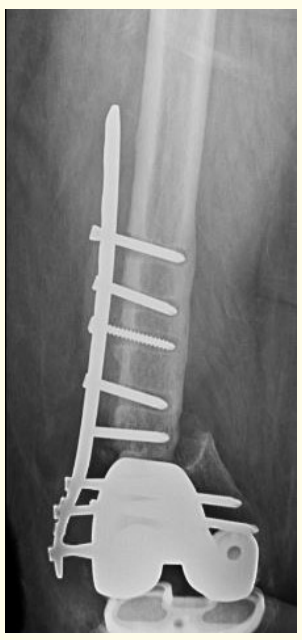

Figure 1e

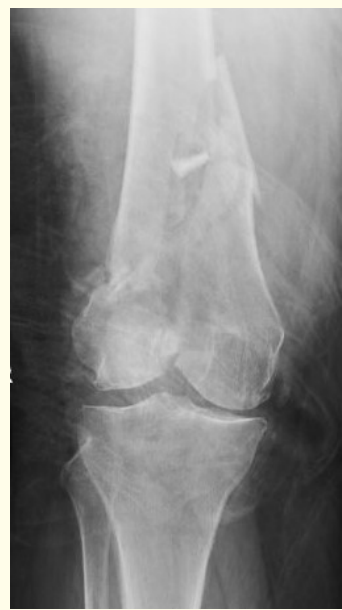

Figure 2a

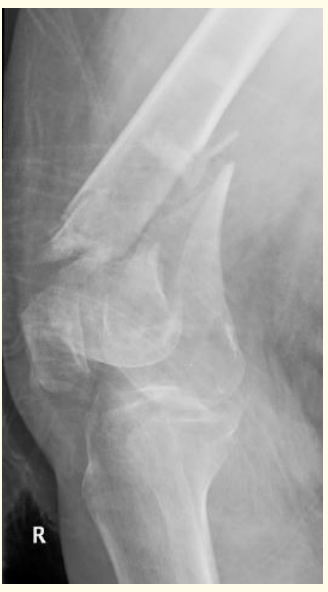

Figure 2b

Figure 1: Aseptic non-union with implant failure.

It has been shown that increasing the plate-bone distance decreases the axial and torsional stiffness [15], this and short working length of the plate could be the reasons for loss of fixation and non-union. This is similar to the rate of aseptic loosening reported by Loosen., et al. [16] and Haidukewych., et al. [17] but much less than the number recorded by Tank., et al. [18] who had 11 implant failures out of 67 patients (16\%).

This character of locked plates, one single stable angular construct, is very advantageous in comminuted fractures with osteoporotic bones. In our study, we fixed 24 distal femur fractures where the BMD was abnormal, all cases united after first intervention (Figure 2).

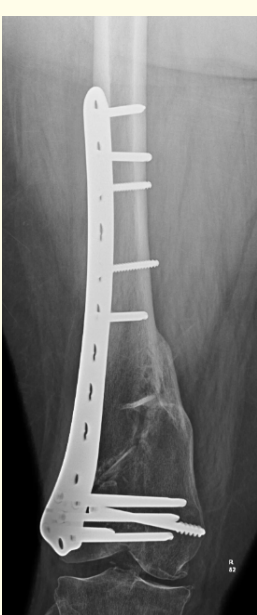

Figure 2c 


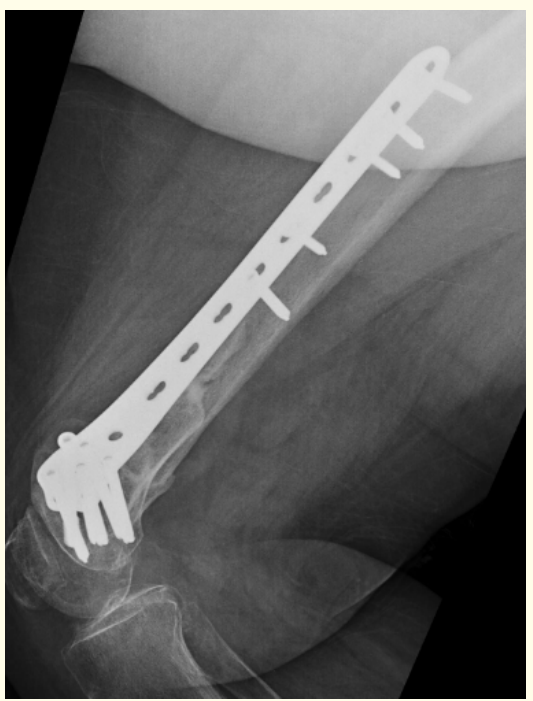

Figure 2d

Figure 2: Comminuted fracture with osteoporotic bone.

Comminution at the fracture site became evident after reduction of the fracture and restoration of length, it was an intraoperative decision to add or not bone substitute to fill the gap to facilitate bone union. In the current study, 10 cases received bone substitutes out of 21 comminuted fractures, but the union time did not show a substantially significant difference when compared with the remaining 11 cases where no gap filling was done. Also, these comminuted fractures achieved union after the index operation (Figure 3). In contrast to locked plating, cement augmentation or bone graft is frequently needed when conventional plates were used to fix distal femur fractures with comminution and osteoporosis [19].

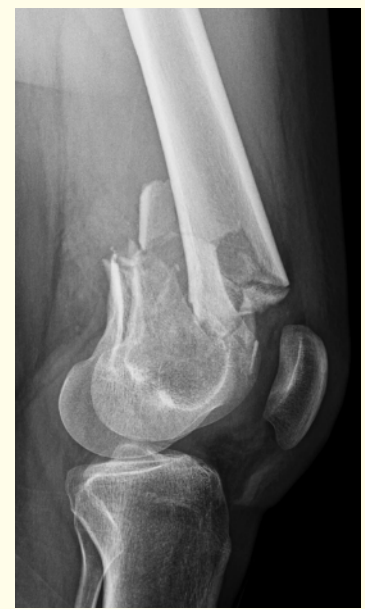

Figure 3a

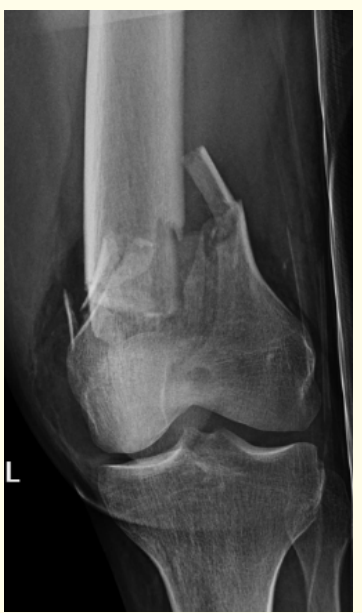

Figure 3b

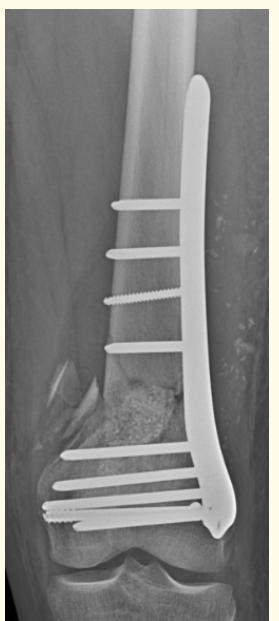

Figure 3c

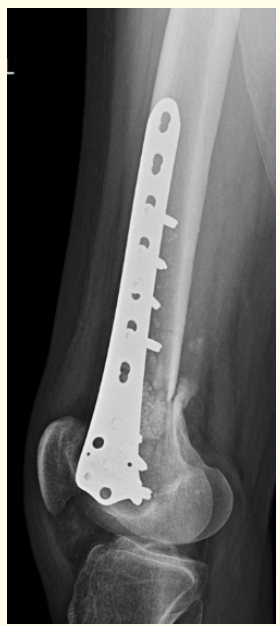

Figure 3d 


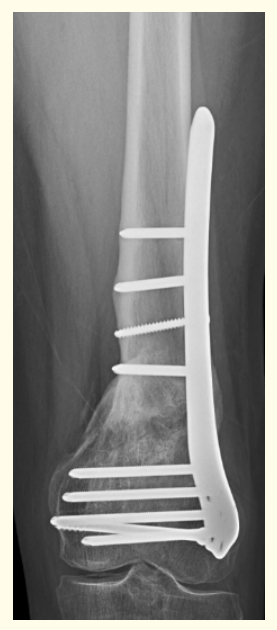

Figure 3e

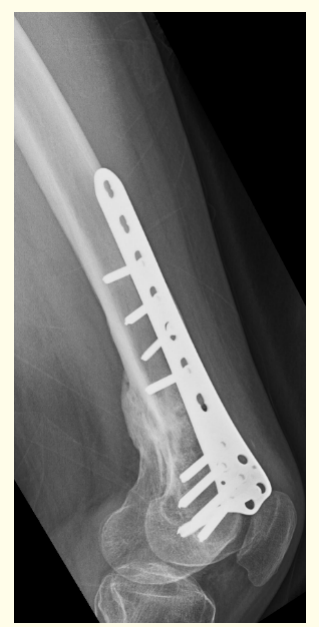

Figure 3f

Figure 3: Union in comminuted fracture with the fracture gap filled with bone substitute.

Gardner., et al. [20] reported that non-union of distal femur occurs most often after open and comminuted fractures. It would be expected that if we added autogenous bone graft to fill the gaps in comminuted fractures, the time to union would be shorter than in those cases where nothing was added to supplement healing. However, the use of locked plating saved time and helped us to avoid donor site morbidity associated with harvesting iliac bone graft.

Our results regarding union of comminuted fractures are consistent with the conclusions of Hierholzer., et al. [21] who found that locked plates had a lower incidence of non-union when used to stabilize distal femur fractures.

Peri-prosthetic distal femoral fractures are more frequently reported recently with the increasing number of knee joint replace- ment surgeries and improved patient's activity post-arthroplasty. The current review included 12 peri-prosthetic fractures, all fixed by the lateral locked distal femoral plate. All fractures united after the first operation except 2 cases went to non-union, one septic and the other was aseptic with construct failure, with union rate of $83 \%$. This mimics the reported rate of union in the study of Ricci., et al. [22] which reached $86 \%$ and also similar to excellent results by Rab and Davis [23]. When conventional plates were used, Figgie., et al. [24] reported 50\% non-union rate in 10 supracondylar femoral peri-prosthetic fractures.

Intramedullary nails (IMN) showed a high rate of malalignment [25]. Biomechanical studies proved that IMN can resist varus stress better than locked plates [26], this difference was clinically insignificant [27]. The use of IMN for distal femoral peri-prosthetic fractures was restricted by the distal bone stock available with the size and position of the femoral component notch [28]. On the contrary, Streubel., et al. [29] showed that the extremely distal periprosthetic supracondylar femoral fractures were successfully fixed by laterally applied locked plates.

Considering the postoperative protocol following the use of locked plates to fix distal femur fractures, we intended to be more careful. Unrestricted active and passive motions were allowed from the second postoperative day, but weight bearing was increased gradually starting after three weeks keeping in mind each patient's general condition. Full weight bearing was allowed only after radiological evidence of bone bridging. Similar protocol was followed by Vink., et al. [30] but they allowed earlier partial weight bearing before three weeks. Also, Loosen., et al. [16] in their review of distal femur fractures in geriatric patients they permitted immediate weight bearing only for 3 out of 50 patients (6\%). However, Poole., et al. [31] allowed immediate full weight bearing as tolerated for $84 \%$ of their patients. Four fractures fail to unite but the rate of clinical and radiological union was 95\%.

The overall function of the patients in the current study was assessed using Pritchett score. It depended mainly on evaluation of the knee range of motion and the presence of residual deformity or pain. Poor results in 6 patients with a reduced and painful range of motions (less than 75 degrees) was attributed to their preoperative state. Five of them had total knee replacement (TKR) followed by peri-prosthetic fracture and the patients reported that there was pain and partial limitation of knee motion before their femur fractures. The remaining patient had originally advanced osteoarthritis of the knee preceding the distal femur fracture.

We achieved overall good results in $70 \%$ of our cases. Similar good results were reported by Vink., et al. [28] and Rademakers., 
et al. [32] who showed that knee function could improve for up to one year after surgery.

\section{Limitations of the Study}

Our study is retrospective and included groups of patients with different age and bone quality. The study sample of 34 patients after exclusion of 7 cases, is relatively small and the results could be more informative if the use of locked plates was compared with the recent designs of locked nails used for distal femur fracture fixation.

\section{Conclusion}

The overall results in this study strongly support the use of locked distal femoral plates for fixation of various patterns of distal femoral fractures particularly in presence of osteoporosis and comminution.

\section{Conflict of Interest}

Authors declare no conflict of interest either financial or in any other means.

\section{Bibliography}

1. Orozco R., et al. "Atlas of Internal Fixation of Fractures of Long Bones". Berlin, Heidelberg: Springer (2000): 201-226.

2. Crist BD., et al. "Treatment of acute distal femur fractures". Orthopedics 31.7 (2008): 681-690.

3. Hoffmann MF., et al. "Clinical outcomes of locked plating of distal femoral fractures in a retrospective cohort". Journal of Orthopaedic Surgery and Research 27.8 (2013): 43.

4. Ehlinger M., et al. "Distal femur fractures, Surgical techniques and a review of the literature". Orthopaedics and Traumatology: Surgery and Research 99.3 (2013): 353-360.

5. Nayak RM., et al. "Minimally invasive plate osteosynthesis using a locking compression plate for distal femoral fractures". Journal of Orthopaedic Surgery 19.2 (2011): 185-190.

6. Henry SL., et al. "Management of supracondylar fractures of the femur with the GSH intramedullary nail: preliminary report". Contemporary Orthopaedics 22.6 (1991): 631-640.

7. Grass R., et al. "Retrograde locking nail osteosynthesis of distal femoral fractures with the distal femoral nail (DFN)". Der Unfallchirurg 105.4 (2002): 298-314.
8. David SM., et al. "Comparative biomechanical analysis of supracondylar femur fracture fixation: locked intramedullary nail versus 95-degree angled plate". Journal of Orthopaedic Trauma 11.5 (1997): 344-350.

9. Kregor PJ., et al. "Distal femoral fracture fixation utilizing the Less Invasive Stabilization System (L.I.S.S.): the technique and early results". Injury 32 (2001): SC 32-47.

10. Weight $M$ and Collinge C. "Early results of the less invasive stabilization system for mechanically unstable fractures of the distal femur (AO/OTA types A2, A3, C2, and C3)". Journal of Orthopaedic Trauma 18.8 (2004): 503-508.

11. Muller ME., et al. "The comprehensive classification of fractures of long bones". 3rd edition. New York, NY: Springer (1991): 118-150.

12. Gustilo RB and Anderson JT. "Prevention of infection in the treatment of one thousand and twenty-five open fractures of long bones: retrospective and prospective analyses". Journal of Bone and Joint Surgery 58 (1976): 453-458.

13. Pritchett JW. "Supracondylar Fractures of the Femur". Clinical Orthopaedics and Related Research 184 (1984): 173-177.

14. Vallier HA., et al. "Failure of LCP condylar plate fixation in the distal part of the femur. A report of six cases". Journal of Bone and Joint Surgery 88.4 (2006): 846-853.

15. Kubiak EN., et al. "The evolution of locked plates". Journal of Bone and Joint Surgery 88.4 (2006): 189-200.

16. Loosen A., et al. "Surgical Treatment of Distal Femur Fractures in Geriatric Patients". Geriatric Orthopaedic Surgery and Rehabilitation 10 (2019): 1-5.

17. Haidukewych G., et al. "Results of polyaxial locked-plate fixation of periarticular fractures of the knee". Journal of Bone and Joint Surgery 89.3 (2007): 614-620.

18. Tank JC., et al. "Early Mechanical Failures of the Synthes Variable Angle Locking Distal Femur Plate". Journal of Orthopaedic Trauma 30.1 (2016): e7-e11.

19. Bezwada HP., et al. "Periprosthetic supracondylar femur fractures following total knee arthroplasty". The Journal of Arthroplasty 19.4 (2004): 453-458.

20. Gardner MJ., et al. "Open reduction and internal fixation of distal femur non-unions: Long term functional outcome following a treatment protocol". Journal of Trauma 64 (2008): 434-438.

21. Hiertholzer C., et al. "Outcome analysis of retrograde nailing and less invasive stabilization system in distal femur fractures: a retrospective analysis". Indian Journal of Orthopaedics 45 (2011): 243-250. 
22. Ricci WM., et al. "Locked plates combined with minimally invasive insertion technique for the treatment of periprosthetic supracondylar femur fractures above a total knee arthroplasty". Journal of Orthopaedic Trauma 20.3 (2006): 190-196.

23. Raab GE., et al. "Early healing with locked condylar plating of periprosthetic fractures around the knee". The Journal of Arthroplasty 20.8 (2005): 984-989.

24. Figgie MP., et al. "The results of treatment of supracondylar fracture above total knee arthroplasty". The Journal of Arthroplasty 5.3 (1990): 267-276.

25. El-Kawy S., et al. "Retrograde femoral nailing in elderly patients with supracondylar fracture femur; is it the answer for a clinical problem?" International Orthopaedics 31.1 (2007): 83-86.

26. Bong MR., et al. "Comparison of the LISS and a retrograde-inserted supracondylar intramedullary nail for fixation of a periprosthetic distal femur fracture proximal to a total knee arthroplasty". The Journal of Arthroplasty 17.7 (2002): 876-881.

27. Large TM., et al. "Locked plating of supracondylar periprosthetic femur fractures". The Journal of Arthroplasty 23.6 (2008): 115-120.

28. Currall VA., et al. "Retrograde nailing for supracondylar fracture around total knee replacement: a compatibility study using the Trigen supracondylar nail". Knee 14.3 (2007): 208-211.

29. Streubel PN., et al. "Are extreme distal periprosthetic supracondylar fractures of the femur too distal to fix using a lateral locked plate?" Journal of Bone and Joint Surgery 92.4 (2010): 527-534.

30. Virk JS., et al. "Distal Femur Locking Plate: The Answer to All Distal Femoral Fractures". Journal of Clinical and Diagnostic Research 10.10 (2016): RC01-RC05.

31. Poole WEC., et al. "'Modern' distal femoral locking plates allow safe, early weight-bearing with a high rate of union and low rate of failure: five-year experience from a United Kingdom major trauma center". Bone and Joint Surgery 99-B.7 (2017): 951-957.

32. Rademakers MV., et al. "Intra-articular fractures of the distal femur: a long-term follow-up study of surgically treated patients". Journal of Orthopaedic Trauma 18.4 (2004): 213-219.

\section{Assets from publication with us}

- Prompt Acknowledgement after receiving the article

- Thorough Double blinded peer review

- Rapid Publication

- Issue of Publication Certificate

- High visibility of your Published work

Website: www.actascientific.com/

Submit Article: www.actascientific.com/submission.php Email us: editor@actascientific.com

Contact us: +919182824667 